\title{
Correction to : Estimating Measurement Uncertainty on Stress-Strain Curves from SHPB
}

\author{
D. Brizard ${ }^{1} \cdot$ S. Ronel ${ }^{1} \cdot$ E. Jacquelin ${ }^{1}$
}

(C) Society for Experimental Mechanics 2020, corrected publication 2021

Equation (12) in the original article [1] gives the strain from the measured voltage on a full bridge configuration of the four strain gages. The full-bridge is intended to measure tension-compression strain (type III full-bridge), the correct equation is therefore [2]

$V_{r}(t)=\frac{e(t)}{V_{\mathrm{ex}}}$
$\epsilon_{\mathrm{III}}(t)=\frac{2 V_{r}(t)}{\kappa\left[(1+v)-V_{r}(t)(1-v)\right]}$

and not

$\epsilon_{\mathrm{II}}(t)=\frac{2 V_{r}(t)}{\kappa(1+v)}$

which corresponds to a type II full-bridge for bending measurements (see table 12.2 in [2]). $\kappa$ is the gage factor, $V_{r}$ the bridge output and $V_{\mathrm{ex}}$ the excitation voltage.

The online version of the original article can be found at https://doi.org/10.1007/s11340-017-0260-8.
D. Brizard
denis.brizard@ifsttar.fr
S. Ronel
sylvie.ronel@univ-lyon1.fr
E. Jacquelin
eric.jacquelin@univ-lyon1.fr
1 Univ Lyon, University Claude Bernard Lyon 1, IFSTTAR, LBMC UMR_T9406, F69622, Lyon, France

The measurement uncertainty on the strain was given as (see equation (13) in [1]):

$$
\begin{aligned}
{\left[\frac{u\left(\epsilon_{\mathrm{II}}(t)\right)}{\epsilon_{\mathrm{II}}(t)}\right]^{2}=} & {\left[\frac{u(\kappa)}{\kappa}\right]^{2}+\left[\frac{u(v)}{1+v}\right]^{2} } \\
& +\left[\frac{u\left(V_{\mathrm{ex}}\right)}{V_{\mathrm{ex}}}\right]^{2}+\left[\frac{u(e(t))}{e(t)}\right]^{2}
\end{aligned}
$$

which holds only in case of type II full-bridge (Equation 3). For type III full-bridge, from Equation 2 and the rules for expressing uncertainty (see [3]), the uncertainty in the strain is:

$$
\begin{aligned}
{\left[\frac{u\left(\epsilon_{\mathrm{III}}\right)}{\epsilon_{\mathrm{III}}}\right]^{2}=} & {\left[\frac{\left(1+V_{r}\right) v}{(1+v)-V_{r}(1-v)}\right]^{2}\left[\frac{u(v)}{v}\right]^{2}+\left[\frac{u(\kappa)}{\kappa}\right]^{2} } \\
& +\left[\frac{1+v}{(1+v)-V_{r}(1-v)}\right]^{2}\left[\frac{u(e)}{e}\right]^{2} \\
& +\left[\frac{1+v}{(1+v)-V_{r}(1-v)}\right]^{2}\left[\frac{u\left(V_{\mathrm{ex}}\right)}{V_{\mathrm{ex}}}\right]^{2}
\end{aligned}
$$

A first order asymptotic approximation of Equation 2 gives

$\epsilon_{\text {III }}=\frac{2 V_{r}}{\kappa(1+v)}\left(1+o\left(V_{r}\right)\right)$

Finally, the order of magnitude of $V_{r}$ during the experiments was of a few thousandth. The consequences of the bridge type error on the results (i.e. strain and measurement uncertainty on the strain) are therefore negligible. 


\section{References}

1. Brizard D, Ronel S, Jacquelin E (2017) Estimating measurement uncertainty on stress-Strain curves from SHPB. Exp Mech 57(5):735-742

2. Watson RB (2008) Bonded Electrical Resistance Strain Gages. In: William N. Sharpe, editor, Springer Handbook of Experimental Solid Mechanics, Springer Handbooks, pages 283-334. Springer US, Boston, MA
3. Farrance I, Frenkel R (2012) Uncertainty of measurement: a review of the rules for calculating uncertainty components through functional relationships. The Clinical Biochemist Reviews 33(2):49-75

Publisher's Note Springer Nature remains neutral with regard to jurisdictional claims in published maps and institutional affiliations. 